\title{
Security against Sample Pair Steganalysis in Eight Queens Data Hiding Technique
}

\author{
Abhishek Bansal \\ Assistant Professor, Indira Gandhi National Tribal University, Amarkantak, M.P \\ E-mail: bansalabhishek28@gmail.com \\ Sunil K. Muttoo and Vinay Kumar \\ Assoiciate Professor, Department of Computer Science, University of Delhi, Delhi \\ Professor, School of Information Technology, VIPS, Delhi \\ E-mail: skmuttoo@cs.du.ac.in, vinay5861@gmail.com
}

\begin{abstract}
There are many steganalysis methods, which can estimate length of a message embedded in least significant bits. It may be embedded either in spatial domain or in frequency domain. The well known approaches are Chi - Square test, RS steganalysis and Sample Pair steganalysis. Many commercial steganographic programs are based on LSB method. It is important to ensure undetectablity of a hidden message in a carrier. We present an analysis of steganographic security on data hiding approach using eight queen solutions. In this approach, relationship between message bytes and 8-queen solutions is embedded in the cover. Further, we propose a new approach to adjust the statistical properties of the cover image in such a way that the steganalyst may not be able to detect the presence of hidden message. The proposed approach is tested using steganalysis tool STEGEXPOSE and the experimental results found are within acceptable range.
\end{abstract}

Index Terms - Steganography, Eight-Queen's problem, sample pair steganalysis, RS steganalysis, Least Significant Bit (LSB) embedding and pseudo randomization, Detectability.

\section{INTRODUCTION}

The purpose of digital steganography is to conceal information inside a digital cover. A digital cover may be image, maps, audio or video files. The important requirements [7] for any steganographic methods are imperceptibility, capacity, and robustness. However, it is difficult for any steganographic method to simultaneously satisfy all of the three requirements. In the recent years, many steganographic methods are developed in which these steganographic requirements are finely balanced. These methods are mainly divided in two groups: spatial domain $[3,6,18,19,22]$ and frequency domain [12, 13, 14]. In spatial domain methods, the least significant bit (LSB) embedding are extensively used to hide secret data because it is easy to implement and it offers high embedding capacity. In this approach, raster scans $[6,19]$ and random scans $[18,20]$ have been adopted to hide the secret data in the visited pixel. For security concern, the random scan embedding is preferred over the raster scan embedding. Muttoo et al [4, 18] proposed a data hiding method based on eight queen solutions. This method helps in randomizing the bit selection in a cover image for hiding purpose. The hiding methodology is based on hiding relationship between message bytes and 8-queen solutions in the cover rather than the message directly. Kumar et al [22] further proposed data hiding technique based on Inter-Block Difference in Eight Queens Solutions and LSB Substitution. The technique provides a better security and high embedding capacity. Another improved technique based on eight queen solutions is proposed by Bansal et al [3]. The method is based on pixel mapping and eight queens' solutions for embedding high payload of secret information. Bansal et al [2] also proposed data hiding technique for improving the security in exploiting modification direction method using knight tour in $8 \times 8$ block of the cover. The method achieves better security using eight queens' solutions and knight path.

Numbers of steganalysis methods have been developed based on statistical properties of image such as ChiSquare test, RS steganalysis and Sample Pair steganalysis. Fridrich et al [9] proposed RS steganalysis method based on the partition of image pixels into three groups: Regular (R), Singular (S) and Unusable groups (U). Fridrich proposed heuristic assumption that the RS ratio of a natural image should satisfy the certain rule. Dumitrescu et al. [8] proposed analytical proof of an observation made by Fridrich et al. [9]. This method can detect the existence of hidden message that are randomly embedded into the least significant bits of natural images. It is based on state transition among various statistical subsets of an image. Experimental result claims that if the embedding ratio is more than 0.03 bit per pixel then this method is very efficient to estimate the length with relatively high precision. Andrew D. Ker [10] performs statistically accurate evaluation of the reliability of image steganalysis method. It mainly focuses on the RS and SP steganalysis for detection of LSB steganography in natural image and also suggests some improvement. Andrew D. Ker [11] also proposed another LSB based 
steganalysis technique using structural steganalysis which gives the most sensitive detectors for standard two LSB replacements.

Most of the steganalysis methods discussed above are based on some statistical hypothesis. We need to develop such steganographic systems, which are statistically robust. In this paper, the security strength of data hiding technique [18] are analyzed with respect to a statistical change and then some enhancement are proposed to make the method more secure and robust against various steganalysis.

The paper is organized in seven sections. Sample pair steganalysis is briefly introduced in Section 2. Section 3 describes data hiding technique using eight queen solutions [18]. The Section 4 of this paper deals with the security analysis of data hiding technique based on eight queen solutions and proposed security enhancement. Experimental results are presented in the Section 5. The section 6 presents the steganalysis using STEGEXPOSE tool. Finally, the paper is concluded in the Section 7.

\section{SAMPLE PAIR ANALYSIS}

Dumitrescu et al. [20] proposed sample pair steganalysis technique based on statistical assumption to detect LSB steganography in continuous-tone natural image. This assumption is very sensitive to LSB embedding and the change in the identity can quantify the length of the embedding message. In this method, we take $\mathrm{P}(\mathrm{u}, \mathrm{v})$ as the set of pixel pairs, where $\mathrm{u}$ and $\mathrm{v}$ are the values of two adjacent horizontal pixel i.e. $0<\mathrm{u}<2^{\mathrm{b}}-1$, $0<\mathrm{v}<2^{\mathrm{b}}-1$. Here $\mathrm{b}$ is the number of bits to represent each pixel in the image. Let $D_{n}$ be the subset of $P$ that consist of pixel pairs of the form $(u, u+n)$ or $(u+n, n)$, i.e. the two values differ exactly by $n$, where $n$ is a fixed integer and $0<n<2^{b}-1$. Since the embedding effect only the LSB, there is another sub multi-set $\mathrm{C}_{\mathrm{m}}$ of $\mathrm{P}$ that consists of the pixel pairs whose value differ by $\mathrm{m}$ in the first $(\mathrm{b}-1)$ bits. For each integer $\mathrm{m}$ and $0 \leq \mathrm{m} \leq 2^{\mathrm{b}-1}-1$, $\mathrm{C}_{\mathrm{m}}$ is define as

$$
\mathrm{C}_{\mathrm{m}}=\{(\mathrm{u}, \mathrm{v}) \in \mathrm{P} \mid \mathrm{u} / 2-\mathrm{v} / 2=\mathrm{m} \text { or } \mathrm{v} / 2-\mathrm{u} / 2=\mathrm{m}\}
$$

Here the multi-sets $\mathrm{D}_{\mathrm{n}}$ forms one partition of $\mathrm{P}$ and the multi-sets $C_{m}$ forms another partition of $P$. It is obvious that $\mathrm{D}_{2 \mathrm{~m}}$ is contained in $\mathrm{C}_{\mathrm{m}}$. In fact if $(\mathrm{u}, \mathrm{v})$ is a pair in $D_{2 m}$ then both $u$ and $v$ are either even or odd. But this is not true for $\mathrm{D}_{2 \mathrm{~m}+1}$. The pixel pairs of $\mathrm{D}_{2 \mathrm{~m}+1}$ are shared between $\mathrm{C}_{\mathrm{m}}$ and $\mathrm{C}_{\mathrm{m}+1}$. We partition $\mathrm{D}_{2 \mathrm{~m}+1}$ into two multisets $\mathrm{X}_{2 \mathrm{~m}+1}$ and $\mathrm{Y}_{2 \mathrm{~m}+1}$, where

$$
\begin{gathered}
\mathrm{X}_{2 \mathrm{~m}+1}=\mathrm{D}_{2 \mathrm{~m}+1} \cap \mathrm{C}_{\mathrm{m}+1}, \\
\mathrm{Y}_{2 \mathrm{~m}+1}=\mathrm{D}_{2 \mathrm{~m}+1} \cap \mathrm{C}_{\mathrm{m}},
\end{gathered}
$$

for $0<\mathrm{m}<2^{\mathrm{b}-1}-2$. Both $\mathrm{X}_{2 \mathrm{~m}+1}$ and $\mathrm{Y}_{2 \mathrm{~m}+1}$ contain pairs ( $\mathrm{u}$, v) that differ by $2 \mathrm{~m}+1(|\mathrm{u}-\mathrm{v}|=2 \mathrm{~m}+1)$. Those pairs with larger even component are in $\mathrm{X}_{2 \mathrm{~m}+1}$ and those with larger odd components are in $\mathrm{Y}_{2 \mathrm{~m}+1}$.
- $\mathrm{X}_{2 \mathrm{~m}+1}$ is the set of pair (u, v) $\in \mathrm{P}$ such that $\mathrm{v}$ is even and $\mathrm{u}<\mathrm{v}$, or $\mathrm{v}$ is odd and $\mathrm{u}>\mathrm{v}$.

- $\mathrm{Y}_{2 \mathrm{~m}+1}$ is the set of pairs $(\mathrm{u}, \mathrm{v}) \in \mathrm{P}$ such that $\mathrm{v}$ is even and $\mathrm{u}>\mathrm{v}$, or $\mathrm{v}$ is odd and $\mathrm{u}<\mathrm{v}$.

The important statistical assumption proposed for natural image is

$$
\left|\mathrm{X}_{2 \mathrm{~m}+1}\right| \approx\left|\mathrm{Y}_{2 \mathrm{~m}+1}\right|
$$

Table 1. Estimated Length of Hidden Message on Different Natural Image using SP Method

\begin{tabular}{|c|c|c|c|}
\hline Image & $\left|\mathbf{X}_{\mathbf{2 m + 1}}\right|$ & $\left|\mathbf{Y}_{\mathbf{2 m + 1}}\right|$ & $\begin{array}{c}\text { Estimate } \\
\text { payload (p) }\end{array}$ \\
\hline Lena.bmp & 87337 & 87922 & 0.0277 \\
\hline Baboon.bmp & 94960 & 94515 & -0.0648 \\
\hline Pepper.bmp & 84745 & 87988 & 0.0484 \\
\hline Pills.tif & 91336 & 90803 & -0.0376 \\
\hline Bear.tif & 86937 & 88731 & 0.0838 \\
\hline Koala.png & 92031 & 92912 & 0.0954 \\
\hline Fishingboat.bmp & 29751 & 29251 & -0.0789 \\
\hline Pentagon.bmp & 30427 & 30929 & 0.0184 \\
\hline
\end{tabular}

This is because natural images are isotropic in terms of the gradient of intensity function, i.e., the gradient of the intensity function in any direction has equal probability. Based on the above statistical results, the following quadratic equation is derived.

$$
\frac{\left(\left|C_{m}\right|-\left|C_{m+1}\right|\right) p^{2}}{4}-\frac{\left(\left|D_{2 m}^{\prime}\right|-\left|D_{2 m+2}^{\prime}\right|\right)+2\left|Y_{2 m+1}^{\prime}\right|-2\left|X_{2 m+1}^{\prime}\right| p}{2}+
$$

Here, $C_{m}, C_{m+1}, D_{2 m}$ and $D_{2 m+1}$ are different multi-set of $\mathrm{P}$ and $|*|$ represents the cardinality of the multi-set $*$ in the embedded image. The result $\mathrm{p}$ of quadratic equation (2) will estimate the length of the embedded message. The minimum value of $p$ is considered the length of hidden message into the cover. Table 1 shows various statistical results on natural images. It shows that the estimated length of hidden message from equation (2) is approximately zero.

\section{DATA HIDING APPROACH USING 8 QUEEN'S SOLUTION}

Data hiding approach based on eight queen solutions is proposed by Muttoo et al [18] in which 8-queens solutions are extracted by placing 8 non-attacking queens on $8 \times 8$ chessboard. In $8 \times 8$ chessboard, the number of distinct 8 queens' solutions is $92[17,21]$. The proposed approach utilizes the feature of 92 different patterns on 8 $\times 8$ block by matching with the secret message. In this procedure, the cover image is divided into blocks of $8 \times 1$ bytes and then block is masked with different solutions of 
the 8-queens problem. Bits from the block are collected corresponding to the 8 -queen solution to make a 7 bit string, which gives a number in the range of 0 to 127 . If a bit string, corresponding to the 8-queens solutions, matches with ASCII code of the first character from message, the corresponding solution number of the 8queens problem is encrypted using $\mathrm{RC} 4$, and the cipher is stored in first block of the cover. This encrypted value works as key. The solution number corresponding to next character is XORED with the key and the resultant value is embedded in the LSB of next block.

In this method, the hiding methodology is based on the hiding relationship between message bytes and 8 -queen solutions rather than the message directly. This method also helps in randomizing the bit selection in a cover image for hiding purpose. The goal of this method is to embed secret message in such a way that the detection of secret message becomes difficult.

In the proposed approach, we analyzed the detection of the existence of the hidden message in the stego image using sample pair steganalysis. Table 2 shows the estimated length of hidden message on different stego image. It shows that the length of hidden message is near to payload of the stego image. Thus, we require some enhancement of the method in order to make it more secure and robust against the SP steganalysis method.

\section{SECURITY ANALYSIS}

We have proposed improvement and undetectablity of data hiding technique based on eight queen solutions [18] As illustrated in section 2, the principle of SP steganalysis method is based on the assumption that

$$
\left|\mathrm{X}_{2 \mathrm{~m}+1}\right| \approx\left|\mathrm{Y}_{2 \mathrm{~m}+1}\right|
$$

It is based on statistical characteristic of the cover. The statistical characteristic is the cardinalities of the set $\mathrm{C}_{\mathrm{m}}$, $D_{2 m}, X_{2 m+1}$ and $Y_{2 m+1}$. For each modification pattern $\pi \epsilon$ $\{00,10,01,11\}$, the statistical characteristic are modified with pattern $\pi$ as a result of embedding. This modification changes the statistical characteristic of the cover image and it is measured using equation (2).

Table 2. Estimated Length using Sample Pair Steganalysis on Various Stego Images of Different Payload $(\gamma)$

\begin{tabular}{|c|c|c|c|c|c|c|c|c|c|}
\hline Payload & \multicolumn{3}{|c|}{$\gamma=0.1$} & \multicolumn{3}{|c|}{$\gamma=0.2$} & \multicolumn{3}{|c|}{$\gamma=0.3$} \\
\hline Image & $\left|X_{2 m+1}\right|$ & $\left|Y_{2 m+1}\right|$ & $\begin{array}{c}\text { Estimate } \\
\text { Length }\end{array}$ & $\left|X_{2 m+1}\right|$ & $\left|Y_{2 m+1}\right|$ & $\begin{array}{c}\text { Estimate } \\
\text { Length }\end{array}$ & $\left|X_{2 m+1}\right|$ & $\left|Y_{2 m+1}\right|$ & $\begin{array}{c}\text { Estimate } \\
\text { Length }\end{array}$ \\
\hline Baboon.bmp & 94202 & 95122 & 0.1293 & 93654 & 95524 & 0.2553 & 92660 & 95518 & 0.2537 \\
\hline Lena.bmp & 85996 & 88491 & 0.1128 & 84852 & 89265 & 0.1933 & 84080 & 89674 & 0.2529 \\
\hline Pepper.bmp & 84745 & 87998 & 0.1353 & 83504 & 88711 & 0.2094 & 82799 & 89130 & 0.2488 \\
\hline Pills.tif & 90620 & 91359 & 0.0513 & 89689 & 91934 & 0.1593 & 89340 & 92521 & 0.2102 \\
\hline Bear.tif & 86346 & 89075 & 0.1261 & 85941 & 89420 & 0.1589 & 85098 & 89998 & 0.2154 \\
\hline Koala.png & 91680 & 93456 & 0.1651 & 91224 & 94247 & 0.2447 & 90421 & 94553 & 0.3256 \\
\hline Pentagon.bmp & 30427 & 30929 & 0.1274 & 30260 & 31069 & 0.2037 & 30063 & 31229 & 0.2882 \\
\hline Fishingboat.bmp & 29285 & 29556 & 0.0519 & 29071 & 29789 & 0.1542 & 28496 & 30333 & 0.2847 \\
\hline
\end{tabular}

The simple method to avoid statistical modification is to ensure no embedding in the adjacent pixel pairs that differ in their value by less than 3 . However, this method is open to attack because of known locations. Luo et al. [15] proposed a LSB approach against pixel pair steganalysis method in which the adjustment of statistical characteristic is based on compensation algorithm. The compensation algorithm finds adjacent pixels pair such that difference of their value is $2 \mathrm{~m}+1$, where $0 \leq \mathrm{m} \leq 2^{\mathrm{b}-}$ ${ }^{1}-1$ and $b$ is the number of bits to represent each pixel. Then half stego image is added by 1 for adjustment of statistical change after embedding. Here the compensation area can be chosen at will. Therefore, the sender and receiver must have the knowledge about the locations of the pixel in the image where pixel value is added by 1 so that message can be extracted at the receiver end. This is the main drawback of this approach.

In this paper, we present a noble approach to adjust the statistical properties of a cover image in order to increase robustness against SP steganalysis. We also present an additional security layer to enhance security and privacy of the embedded message.

\section{A. Robust Hiding Method}

In the proposed method, cover image is divided into two groups of $8 \times 1$ pixel blocks. The first group of $8 \times 1$ pixels block is used to hide secret information and the second group contains no secret information. In order to improve the security strength of the hiding algorithm, the second group is utilized to modify the pixel in such a way that the statistical property $\left|\mathrm{X}_{2 \mathrm{~m}+1}\right|$ and $\left|\mathrm{Y}_{2 \mathrm{~m}+1}\right|$ of stego image remains as similar as that of cover image. Therefore, the estimated length of hidden information by SP steganalysis is near to zero. The experimental results shown in Table 2 shows that the value of $\left|\mathrm{Y}_{2 \mathrm{~m}+1}\right|$ is higher than $\left|\mathrm{X}_{2 \mathrm{~m}+1}\right|$. Thus, the result of estimated length of hidden information in stego image is near to the payload in the cover.

The assumption 1 on natural image implies that the gradient in any direction of natural image has equal 
probability. Therefore, we need to adjust such pair in which odd components is larger i.e., decrease the cardinality of $\mathrm{Y}_{2 \mathrm{~m}+1}$. Enhancement to this algorithm is discussed in this paper to to make the approach secure and robust.

Let $\mathrm{P}\left(\mathrm{u}_{\mathrm{i}}, \mathrm{v}_{\mathrm{i}}\right)$ be sample pair of two horizontal adjacent pixels. The sample pair to be modified, should satisfy the condition $\left|u_{i}-v_{i}\right|<\delta$, where $\delta$ is threshold value. Let $\alpha_{m}=$ $\left|\mathrm{Y}_{2 \mathrm{~m}+1}\right|-\left|\mathrm{X}_{2 \mathrm{~m}+1}\right|$, where $0 \leq \mathrm{m} \leq 2^{\mathrm{b}-1}-2$ and $\mathrm{b}$ be the number of bits to represent a pixel in the cover. We need to decrease the cardinality of $\mathrm{Y}_{2 \mathrm{~m}+1}$ in order to make this value almost equal to the cardinality of $\mathrm{X}_{2 \mathrm{~m}+1}$. Here, $\mathrm{Y}_{2 \mathrm{~m}+1}$ is the set of pair $\left(\mathrm{u}_{\mathrm{i}}, \mathrm{v}_{\mathrm{i}}\right) \in \mathrm{P}$ such that

$$
v \text { is even if } u>v \text {, or } v \text { is odd if } u<v \text {. }
$$

In this approach, we consider sample pair $\mathrm{P}\left(\mathrm{u}_{\mathrm{i}}, \mathrm{v}_{\mathrm{i}}\right)$ in which $v_{i}$ is odd, $u_{i}<v_{i}$, and $\left|u_{i}-v_{i}\right|<\delta$. Further, we increase the value $u_{i}$ where, $u_{i}<(255-\delta)$ as

$$
\mathrm{u}_{\mathrm{i}}=\mathrm{u}_{\mathrm{i}}+\left|\mathrm{u}_{\mathrm{i}}-\mathrm{v}_{\mathrm{i}}\right|+1
$$

thus making $\mathrm{u}_{\mathrm{i}}>\mathrm{v}_{\mathrm{i}}$ in the sample pair $\mathrm{P}\left(\mathrm{u}_{\mathrm{i}}, \mathrm{v}_{\mathrm{i}}\right)$. However, $v_{i}$ remains odd therefore, the cardinality of $Y_{2 m+1}$ decreases by one, while the cardinality of $\mathrm{X}_{2 \mathrm{~m}+1}$ increases by one. To satisfy assumption 1 , we need to modify at least $\left(\alpha_{\mathrm{m}} / 2\right)$ sample pairs in the cover image. The stepwise procedure is given below.

\section{B. Algorithm to enhance robustness}

Step 1: Embed the secret message using the proposed method and take a threshold value $\delta$.

Step 2: Find statistical properties of $\left|\mathrm{X}_{2 \mathrm{~m}+1}\right|$ and $\left|\mathrm{Y}_{2 \mathrm{~m}+1}\right|$ using sample pair steganalysis and calculate $\alpha=$ $\left(\left(\left|\mathrm{Y}_{2 \mathrm{~m}+1}\right|-\left|\mathrm{X}_{2 \mathrm{~m}+1}\right|\right) / 2\right)$, where $0 \leq \mathrm{m} \leq 2^{\mathrm{b}-1}-2$, here $\mathrm{b}$ is number of bits to represent pixel in the cover.

Step 3: Find $8 \times 1$ pixel block, which does not contain secret message, and make sample pair with their adjacent pixel such as $\left(\mathrm{u}_{\mathrm{i}}, \mathrm{v}_{\mathrm{i}}\right)$, where $\mathrm{u}_{\mathrm{i}}$ is $\mathrm{i}^{\text {th }}$ pixel value of $8 \times 1$ block and $v_{i}$ is the horizontal right adjacent pixel of $u_{i}$, here $i=1,2, \ldots .8$

Step 4: Let $\mathrm{x}=1$

Step 5: In each $8 \times 1$ pixel block, starting form second block, for sample pair $\left(u_{i}, v_{i}\right), i=2,3,4, \ldots 8$ do the following

If $\left(\bmod \left(\mathrm{v}_{\mathrm{i}}, 2\right)==1\right)$ and $\mathrm{u}_{\mathrm{i}}<\mathrm{v}_{\mathrm{i}}$ then $\mathrm{d}_{\mathrm{i}}=\left|\mathrm{u}_{\mathrm{i}}-\mathrm{v}_{\mathrm{i}}\right|$

If $\mathrm{d}_{\mathrm{i}}<\delta$ and $\mathrm{u}<(255-\delta)$ do the following

Find $r=u_{i}+\left(d_{i}+1\right)$

$\mathrm{u}_{\mathrm{i}}=\mathrm{r}$

$\mathrm{x}=\mathrm{x}+1$

Step 6: While $\mathrm{x}<\alpha$, repeat step 5 for next pixel block

Step 7: Find the statistical properties of $\left|\mathrm{X}_{2 \mathrm{~m}+1}\right|,\left|\mathrm{Y}_{2 \mathrm{~m}+1}\right|$ and estimate hidden message length $\mathrm{p}$ in the stego image.

Step 8: If $\mathrm{p} \geq 0.07$ then calculate $\beta=|| \mathrm{Y}_{2 \mathrm{~m}+1}|-| \mathrm{X}_{2 \mathrm{~m}+1}|| /$ 2

Step 9: $\alpha=\alpha+\beta$ and go to step 4

C. Resuhuffling of 8queen's solution array
The security of data hiding approach using eight queen solutions [18] depends on RC4 encryption. Once the RC4 encryption is cracked, the secret message can be revealed. An 8-queen problem has 92 solutions. All solutions are stored in two dimensional arrays $Q$ of size $92 \times 8$. The solutions of eight queen problem are stored in an array $Q$ as shown in Figure 1. Each character $\mathrm{C}$ from message $\mathrm{M}$ is compared with 7 bit string from Q. If a match is found, then solution number corresponding to next character is XORED with the key and the resultant value is embedded in the LSB of corresponding $8 \times 8$ bits block. First character of $\mathrm{M}$ is compared with 92 solutions from Q. If a match id found then the solution number is considered as a key. It means the key should be any one number from 1 to 92 . It can be easily detected by brute force method. For a high security, we propose the new concept to reshuffle the array of eight queen solutions using key scheduling algorithm for each block.

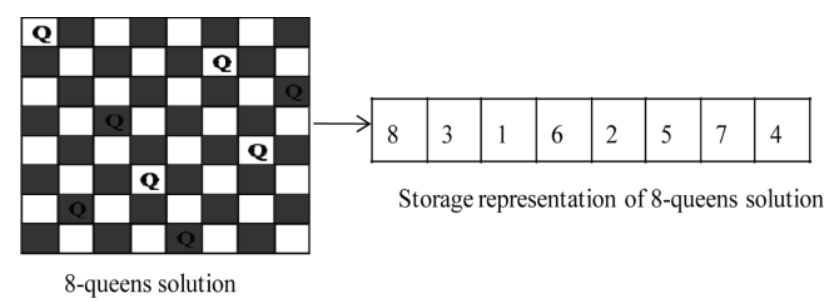

Fig.1 Storage Representation of Eight Queen Solutions

In the key scheduling algorithm (KSA), the array Q is reshuffled with the stego-key [1]. A variable length stego key between 40 to 92 bits is used to initialize a 92-byte state vector $S$ that contains a permutation of all solutions of eight queens' problem. $\mathrm{S}$ is initialized with values from 0 to 91 in ascending order i.e., $\mathrm{S}[0]=0, \mathrm{~S}[1]=1$, $\mathrm{S}[2]=2, \ldots ., \mathrm{S}[91]=91$. Let $\mathrm{T}$ be a temporary vector. If the length of key $\mathrm{K}$ is 92 bits then $\mathrm{S}$ is transferred to $\mathrm{T}$, otherwise length 1 of key is used to initialize $\mathrm{T}$ as below:

$$
\begin{aligned}
& \text { For } \mathrm{i}=0 \text { to } 91 \text { do } \\
& \mathrm{S}[\mathrm{i}]=\mathrm{i} ; \\
& \mathrm{T}[\mathrm{i}]=\mathrm{S}[\mathrm{i} \bmod 1]
\end{aligned}
$$

Then temporary vector $\mathrm{T}$ is used to produce the permutation of $\mathrm{S}$ as below:

$$
\begin{aligned}
& \mathrm{j}=0 \\
& \text { For } \mathrm{i}=0 \text { to } 91 \text { do } \\
& \mathrm{j}=(\mathrm{j}+\mathrm{S}[\mathrm{i}]+\mathrm{T}[\mathrm{i}]) \bmod 92 \\
& \text { Swap }(\mathrm{S}[\mathrm{i}], \mathrm{S}[\mathrm{j}])
\end{aligned}
$$

After this swap, S still contains solutions number from 0 to 91 of all the solutions of eight queens' problem but in different order. This approach is more secure because array $\mathrm{Q}$ is reshuffled for each block. Therefore even if $\mathrm{RC} 4$ encryption is cracked, the secret message cannot be retrieved without knowing the stego key.

\section{EXPERIMENTAL RESULT}

Standard cover images and corresponding stego images 
are presented in Figure 2. We observe that there is no visual difference due to embedding and adjustment of statistical properties in the cover. We carried out experiments with hundreds of never compressed test images from http://www.petitcolas.net/watermarking/ image_database /index.html. All of these test images were converted to $256 \times 256$ pixels size. To assess the visual quality of an image after embedding, we used the Peak-Signal-to-Noise Ratio (PSNR) as the measure of distortion due to data hiding. The PSNR is defined as follows:

$$
\begin{gathered}
P S N R=10 \times \log _{10} \frac{255^{2}}{M S E} d B, \\
M S E=\frac{M S E_{R}+M S E_{G}+M S E_{B}}{3}, \\
M S E_{R}=\sum_{i=0}^{M-1} \sum_{j=0}^{N-1}\left(X_{r}(i, j)-I^{\prime}{ }_{r}(i, j)\right)^{2}
\end{gathered}
$$

where, $\mathrm{X}(i, \mathrm{j})$ and $\mathrm{I}_{\mathrm{r}}(i, \mathrm{j})$ represent the values of the pixel at location $(i, j)$ in the original image and the stego image respectively. $M$ and $N$ represent height and width of the images respectively. The PSNR value of greater than 30 $\mathrm{dB}$ is considered as safe value for retaining the similarity between cover and stego images. In this case, it is hard to distinguish stego images from its corresponding cover image through human eyes. We used MATLAB 9.0 to test the result.
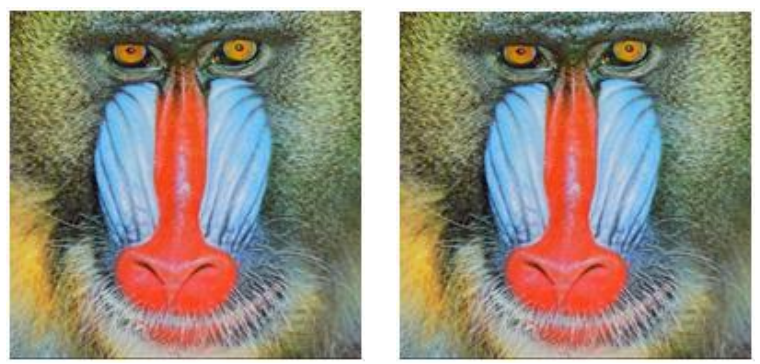

(a)
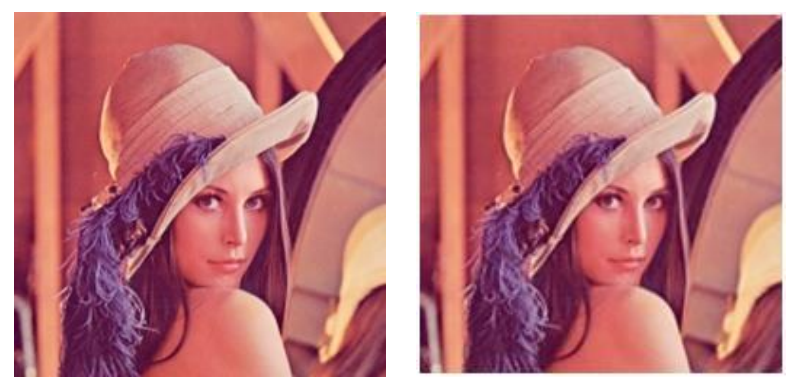

(b)
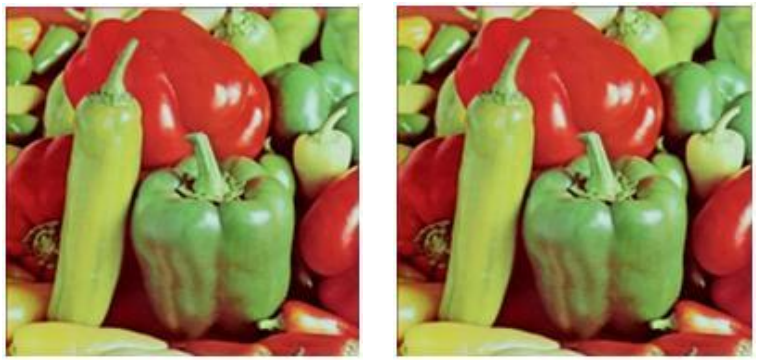

(c)
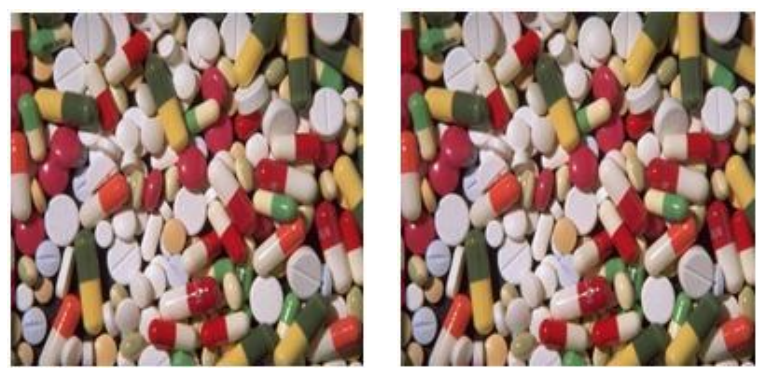

(d)
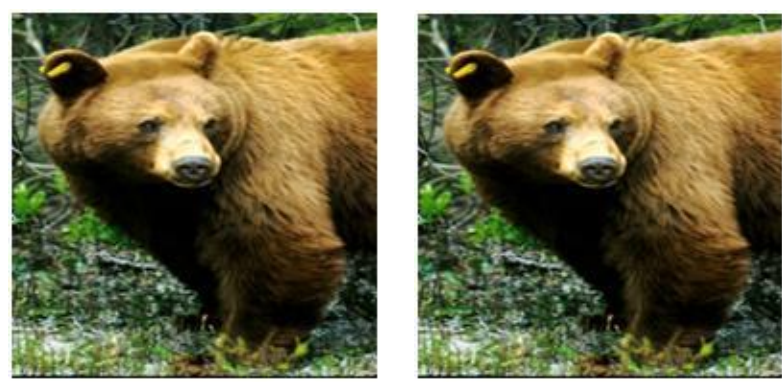

(e)
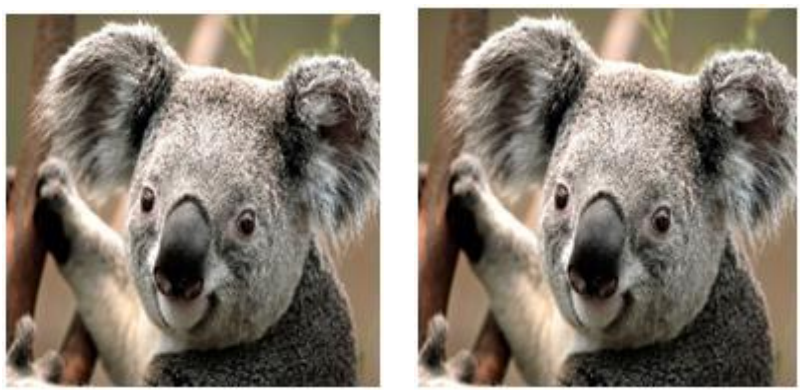

(f)
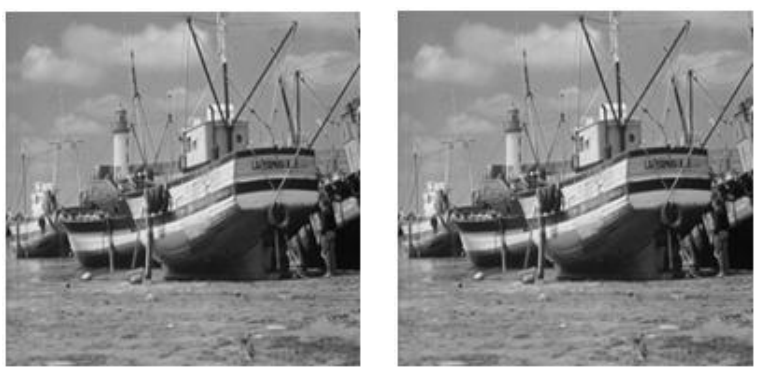

(g) 

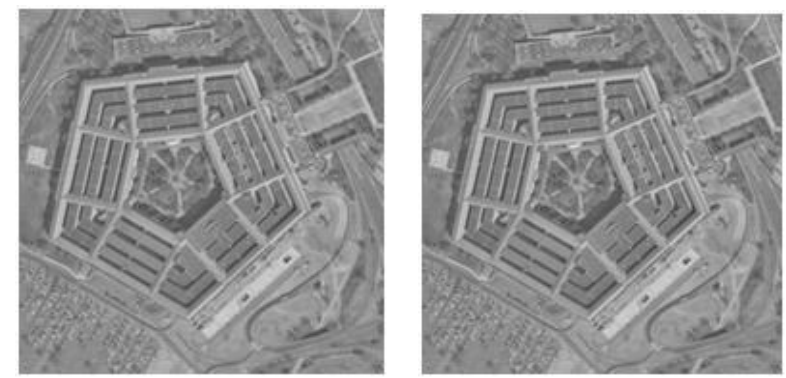

(h)

Fig.2. Cover image and stego image after adjustment of statistical properties at different embedding rate $(\gamma)$. (a) Baboon.bmp and Stego image at $\gamma=0.50$ (b) Lena.bmp and Stego image at $\gamma=0.40$ (c) Peppers.bmp and Stego image at $\gamma=0.40$ (d) Pills.png and stego image at $\gamma=0.30$ (e) Bears.tif and stego image at $\gamma=0.30$ (f) Koala.tif and stego image at $\gamma=0.30(\mathrm{~g})$ Fishingboat.bmp and stego image at $\gamma=0.30$ (h) Pentagon.bmp and stego image at $\gamma=0.20$
We performed statistical analysis using SP steganalysis method on thousands of 24-bit colour and greyscales images. We calculated various statistical properties like $\left|\mathrm{X}_{2 \mathrm{~m}+1}\right|,\left|\mathrm{Y}_{2 \mathrm{~m}+1}\right|$ and estimated payload using sample pair method on different stego images. Some of the results are shown in Table 3.

Table 3. Sample Pair Statistical Properties of Different Stego Images at Threshold $\delta=4$

\begin{tabular}{|c|c|c|c|c|c|c|c|c|c|}
\hline \multirow{3}{*}{ Image } & \multicolumn{9}{|c|}{ Threshold $\delta=4$} \\
\hline & \multicolumn{3}{|c|}{$\gamma=0.1$} & \multicolumn{3}{|c|}{$\gamma=0.2$} & \multicolumn{3}{|c|}{$\gamma=0.3$} \\
\hline & $\left|X_{2 m+l}\right|$ & $\left|Y_{2 m+1}\right|$ & $\begin{array}{l}\text { Estimate } \\
\text { Payload }\end{array}$ & $\left|X_{2 m+1}\right|$ & $\left|Y_{2 m+1}\right|$ & $\begin{array}{l}\text { Estimate } \\
\text { Payload }\end{array}$ & $\left|X_{2 m+l}\right|$ & $\left|Y_{2 m+1}\right|$ & $\begin{array}{l}\text { Estimate } \\
\text { Payload }\end{array}$ \\
\hline Baboon.bmp & 94478 & 94888 & 0.0584 & 94418 & 94849 & 0.0606 & 94407 & 94788 & 0.0530 \\
\hline Lena.bmp & 87614 & 87107 & -0.0235 & 87462 & 86978 & -0.0221 & 87273 & 86995 & -0.0126 \\
\hline Pepper.bmp & 85666 & 87179 & 0.0640 & 85864 & 86675 & 0.0640 & 85483 & 86620 & 0.0466 \\
\hline Pills.tif & 90795 & 91195 & 0.0277 & 90483 & 91169 & 0.0478 & 90419 & 91283 & 0.0586 \\
\hline Bear.tif & 87086 & 88395 & 0.0617 & 86909 & 88168 & 0.0647 & 86980 & 88319 & 0.0624 \\
\hline Koala.png & 92609 & 92961 & 0.0325 & 92466 & 92970 & 0.0459 & 92471 & 93053 & 0.0658 \\
\hline Cat.png & 93533 & 94128 & 0.0681 & 93532 & 94130 & 0.0684 & 95536 & 94126 & 0.0675 \\
\hline Fishingboat.bmp & 29507 & 29358 & -0.0233 & 29379 & 29494 & -0.0185 & 29311 & 29665 & 0.0602 \\
\hline Pentagon.bmp & 30557 & 30800 & 0.0618 & 30524 & 30771 & 0.0618 & 30502 & 30768 & 0.0649 \\
\hline
\end{tabular}

Table 4. PSNR and Embedding Capacity $\gamma$ (bit per pixel) of Different Stego Image at Threshold $\delta=4$

\begin{tabular}{|c|c|c|c|}
\hline \multirow{2}{*}{ Image } & \multicolumn{3}{|c|}{ PSNR } \\
\cline { 2 - 4 } & $\gamma=\mathbf{0 . 1}$ & $\gamma=\mathbf{0 . 2}$ & $\gamma=\mathbf{0 . 3}$ \\
\hline Baboon.bmp & 55.6808 & 52.5865 & 50.6873 \\
\hline Lena.bmp & 54.3215 & 51.8932 & 50.0591 \\
\hline Pepper.bmp & 54.9350 & 51.8206 & 50.0856 \\
\hline Pill.tif & 55.2498 & 52.1032 & 50.3628 \\
\hline Bear.tif & 55.4763 & 52.2163 & 50.0110 \\
\hline Koala.png & 55.0589 & 52.2713 & 50.5242 \\
\hline Fishingboat.bmp & 55.1575 & 52.0292 & 50.3214 \\
\hline Pentagon.bmp & 55.2511 & 52.1171 & 50.6722 \\
\hline
\end{tabular}

The experimental results show that the statistical properties $\left|\mathrm{X}_{2 \mathrm{~m}+1}\right|$ and $\left|\mathrm{Y}_{2 \mathrm{~m}+1}\right|$ are near to each other and the estimated payload $\mathrm{p}$ is below 0.07 of length of hidden message. The SP steganalysis fails to detect presence of hidden information in the cover image. The PSNR results shown in Table 4 confirm that the imperceptibility level of stego image is within acceptable range. The proposed method is tested on different threshold values $\delta$. By comparing the value of PSNR v/s payload for threshold $\delta$
$=2$ to 4 as shown in Figure 3, it is observed that the result of threshold $\delta=2$ are slightly better than others threshold.

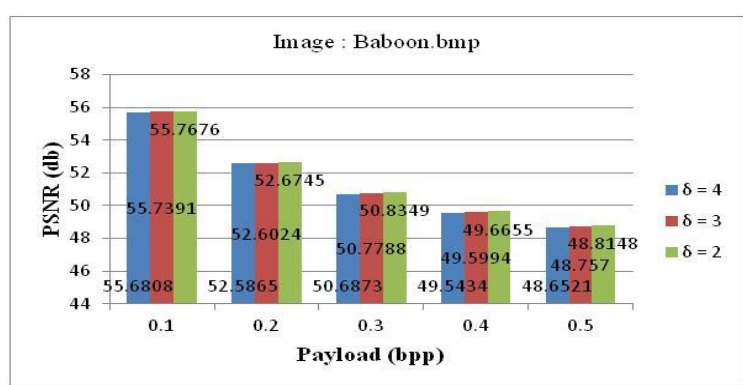

Fig.3. PSNR Vs Payload on Different Threshold of Baboon.bmp

\section{STEGANALYSis}

We used STEGEXPOSE [5] - a steganalysis tool. It is basically based on the fusion technique that consists of popular steganalysis methods: RS analysis, SP analysis, chi-square attack and difference histogram analysis. The experimental results obtained using STEGEXPOSE tool 
on various stego images of proposed approach are shown in Figure 4. The estimated length of hidden message is below 0.07 for different embedding capacity. Therefore, the proposed method is robust against SP steganalysis and STEGEXPOSE tools.

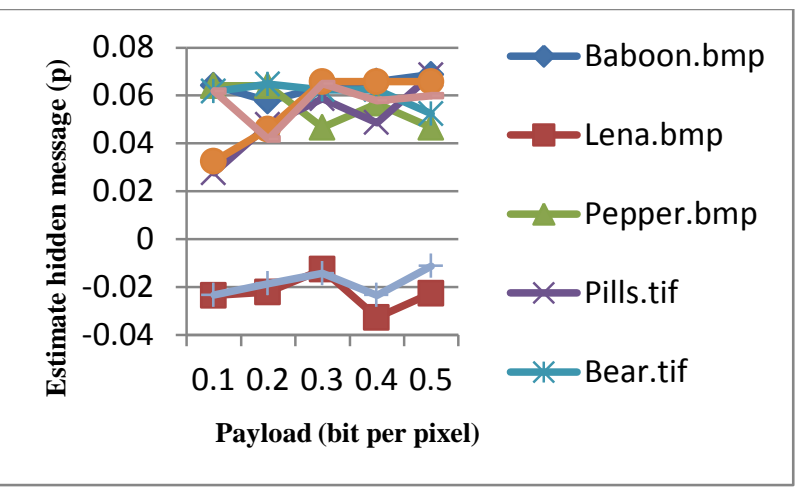

Fig.4. Payload Vs Estimated Length (p) of Hidden Message

\section{CONCLUSION}

In this paper, we discussed the security of data hiding method based on eight queen solutions and its improvement on two different aspects. We analyzed the detection of the existence of the hidden message using sample pair steganalysis and then we proposed enhancement in order to achieve robustness in hiding techniques against sample pair steganalysis. We analyzed the strength of data hiding approach with respect to security and then suggested a new security layer by reshuffling the array of eight queen solutions for each block based on stego-key. The experimental results show that the estimated length of hidden message after adjustment of statistical properties is near to zero. The proposed method is secure and robust against detection of secret message. Some experiments were carried to indentify the maximum payload in a cover and found that the embedding capacity is almost 0.5 bit per pixel on different image. This corroborates that there exists a trade-off between capacity and undetectablity. We will test this approach with other steganalysis tools/methods to improve undetectablity of secret information.

\section{ACKNOWLEDGMENT}

We are very grateful to all those who have been constantly encouraging and providing required resources for such scientific research work besides the regular work which we are doing in our respective departments. We are grateful to our colleagues and seniors for their continuous support and encouragements and to all those who provided suggestions for improvement of this work. We are thankful to Jessica Fridrich, who supplied the SP steganalysis code on her website http://dde.binghamton. edu/download/structural_lsb_detectors.

\section{REFERENCES}

[1] Akgün, M., Kavak, P., \& Demirci, H. (2008). New results on the key scheduling algorithm of RC4. In Progress in Cryptology-INDOCRYPT 2008, Springer Berlin Heidelberg, pp 40-52.

[2] Bansal A., Muttoo S. K., Kumar V. (2015), "Secure Data Hiding Along Randomly Selected Closed Knight Tour", International Journal of Applied Security Research, Taylor \& Francis, vol. 10 no. 1 (In Press).

[3] Bansal A., Muttoo S. K., Kumar V., (2014) Data Hiding approach based on Eight-Queens Problem and Pixel Mapping Method, International Journal of Signal Processing, Vol.7 No. 5, pp 47-58.

[4] Bell, Jordan and Stevens, Brett, (2009), 'A survey of known results and research area for n-queens', Discrete mathematics, Vol. 309, No. 1, pp 1-31.

[5] Boehm, B. (2014). StegExpose-A Tool for Detecting LSB Steganography.arXiv preprint arXiv:1410.6656.

[6] Chan, C. K., \& Cheng, L. M. (2004). 'Hiding data in images by simple LSB substitution'. Pattern recognition, 37(3), pp. 469-474.

[7] Cheddad, A., Condell, J., Curran, K., \& Mc Kevitt, P. (2010). Digital image steganography: Survey and analysis of current methods. Signal processing, 90(3), pp. 727-752.

[8] Dumitrescu, S., Wu, X., \& Memon, N. (2002, June). On steganalysis of random LSB embedding in continuoustone images. In Image Processing. 2002. Proceedings. 2002 International Conference on IEEE, Vol. 3, pp. 641644.

[9] Fridrich, J., Goljan, M., \& Du, R. (2001, October). Reliable detection of LSB steganography in color and grayscale images. In Proceedings of the 2001 workshop on Multimedia and security: new challenges, ACM, New York, NY, USA, pp. 27-30.

[10] Ker, A. D. (2004, June). Quantitative evaluation of pairs and RS steganalysis. In Electronic Imaging 2004, International Society for Optics and Photonics, pp. 83-97

[11] Ker, Andrew D., (2007), "Steganalysis of embedding in two least-significant bits. "Information Forensics and Security, IEEE Transactions on vol. 2. No.1, pp. 46-54.

[12] Kumar, S., \& Muttoo, S. K. (2009, November). Distortionless data hiding based on Slantlet transform. In Multimedia Information Networking and Security, 2009. MINES'09. International Conference on IEEE, Vol. 1, pp. 48-52.

[13] Kumar, S., \& Muttoo, S. K. (2011). Steganography based on contourlet transform. International Journal of Computer Science and Information Security, 9(6), 215.

[14] Kumar, S., \& Muttoo, S. K. (2013). A comparative study of image steganography in wavelet domain. International Journal of Computer Science and Mobile Computing, 2(2), pp. 91-101.

[15] Luo, X., Liu, F., \& Lu, P. (2007). A LSB steganography approach against pixels sample pairs steganalysis. International Journal of Innovative Computing, Information and Control (IJICIC), 3(3), pp. 575-588.

[16] M. Reichling, (1987), "A simplified solution of the $n$ queens problem”, Inform. Process. Lett. 25, pp. 253-255.

[17] Madachy, J. S. (1979). Madachy's Mathematical Recreations. Dover Publications.

[18] Muttoo S. K., Kumar V and Bansal A, (2012) "Secure Data Hiding Using Eight Queens Solutions", International Journal of Information Security \& Privacy, USA, Vol. 6, pp 55-70.

[19] Provos, N., \& Honeyman, P. (2003). Hide and seek: An introduction to steganography. Security \& Privacy, IEEE, 1(3), pp 32-44.

[20] S. Dumitrescu, Wu Xiaolin and Zhe Wang, (2003), "Detection of LSB steganography via Sample Pair 
Analysis", In LNCS, vol. 2578, Springer-Verlang, New York, pp. 355-372.

[21] Steinhaus H., (1999), "Mathematical Snapshots", 3rd Ed. New York: Dover, pp. 29-30.

[22] V. Kumar, Bansal A., Muttoo S. K., (2014), "Data Hiding Method Based on Inter-block difference in Eight queens Solutions and LSB Substitution", International Journal of Information Security \& Privacy, USA, Vol. 8, no. 2, pp. $42-52$.

\section{Authors' Profiles}

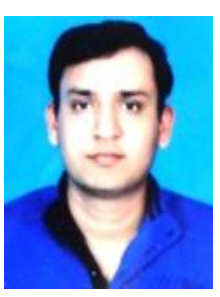

Abhishek Bansal did his MCA from Dr. Bhim Rao Ambedkar University, Agra, India in 2004. He is currently working as a Assistant Professor in the department of Computer Science, Indira Gandhi National Tribal University, M.P. India. $\mathrm{He}$ is pursuing PhD in Information Security from Delhi University, India. He has working experience more than 5 years. His research interests include digital Watermarking, data hiding techniques and image processing.

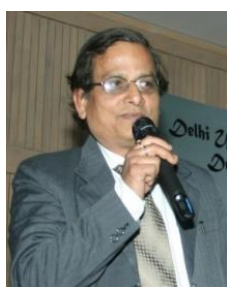

Sunil Kumar Muttoo is Associate Professor in Department of Computer Science, University of Delhi, India. He completed his M. Tech from IIT Kharagpur and Ph.D. from University of Delhi, India. His specialization is coding theory, information hiding.

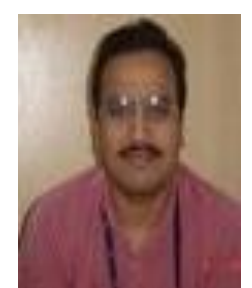

Vinay Kumar is a Professor in Vivekananda Institute of Professional Studies, Delhi. Earlier he worked as Scientist in National Informatics Centre, MoCIT, GOI. He completed his Ph. D. in Computer Science from University of Delhi and MCA from JNU, Delhi. He has authored a book on Discrete Mathematics and contributed many research papers to refereed journals and conferences. His areas of interest are graph algorithm, steganography, data security, data mining and e-governance.

How to cite this paper: Abhishek Bansal, Sunil K. Muttoo, Vinay Kumar,"Security against Sample Pair Steganalysis in Eight Queens Data Hiding Technique", International Journal of Computer Network and Information Security(IJCNIS), Vol.8, No.8, pp.39-46, 2016.DOI: 10.5815/ijcnis.2016.08.05 